\title{
Ovine Catarrhal Fever (Bluetongue): Analysis of Culicoides Species in Seropositive Farms
}

\author{
A. Guercio, P. Di Marco, C. Manno, C. Di Bella, G. Purpari and A. Torina \\ Istituto Zooprofilattico Sperimentale della Sicilia, Palermo, Italy
}

\author{
Keywords: \\ Bluetongue; Culicoides; serotype; Sicily; \\ vectors

\section{Correspondence: \\ Annalisa Guercio. Istituto Zooprofilattico Sperimentale della Sicilia, Via G. Marinuzzi, 3 - 90129 Palermo, Italy. Tel.: \\ +390916565224; Fax: +390916565227; \\ E-mail: annalisa.guercio@izssicilia.it}

Received for publication January 26, 2010

doi:10.1111/j.1865-1682.2010.01122.x

\begin{abstract}
Summary
Bluetongue (BT) is an orbiviral disease of wild and domestic ruminants, mainly sheep. In Sicily, the first Bluetongue outbreak occurred in October 2000; there have been 76 recorded outbreaks so far. The National Surveillance Plan, based on European Union Commission Decision 138/2001/CE, establishes serological and entomological surveys. This plan consists of controls of seronegative cattle, called 'sentry' as indicators for the presence and circulation of virus in defined areas. To check the seroconversions, the regional territory has been subdivided in $400 \mathrm{~km}^{2}$ areas including 58 seronegative cattle, periodically checked by serological tests. All positive sera have been tested to detect the specific serotype by the National Reference Centre for Exotic Diseases (CESME) at the Istituto Zooprofilattico Sperimentale Abruzzo e Molise in Teramo (IZS Teramo). Moreover, entomological surveillance has been implemented in seropositive herds, to investigate the presence of insect vectors belonging to Culicoides genus. The goal of the present communication is to report on the different species of Culicoides found in the farms with Bluetongue virus and to investigate on the probable role of new competent vectors. This paper concerns data analysis of 581 light-trap catches collected in 321 farms from 2003 to 2008 . We observed that $82 \%$ of checked farms were positive for Culicoides spp., and only $10 \%$ of the farms were positive for Culicoides imicola.
\end{abstract}

\section{Introduction}

Bluetongue (BT) is a non-contagious insect-borne viral infection, which occurs in domestic and wild ruminants such as sheep, goats, cattle, buffaloes, deer and other ruminants. Bluetongue virus (BTV) is a double-stranded RNA-virus $( \pm)$ belonging to the Orbivirus genus within the Reoviridae family. The BTV species contains 24 antigenically different recognized serotypes. Not all BTV serotypes exist in the same area. BTV usually produces unapparent infection in cattle, but it causes fatal disease in sheep, deer and wild ruminants. Cattle are important in the endemism of the disease because they show a very prolonged viraemia in the absence of clinical symptoms. They represent the main reservoir of the virus and can be used as 'sentry' in serological control plans. In the other ruminants, the clinical signs range from mild to severe and they vary not only between species but between breeds. Classic clinical signs are: fever, vascular permeability and haemorrhages, erosion of the mucous membranes, facial oedema and cyanosis of the tongue. BTV is transmitted by biting of blood-feeding insect vectors of the genus Culicoides spp. (Diptera: Ceratopogonidae) (Mellor et al., 2000). More than 1200 Culicoides species have been identified in the world. Culicoides imicola subgenus Avaritia is connected with BTV transmission in Europe and Africa, but C. imicola sensu stricto is only in Europe (Linton et al., 2002). The recent BTV diffusion is probably due to the expansion of $C$. imicola in some European countries (Wittmann and Baylis, 2000). In the past, in Italy, this species had never been recorded (Baylis and Mellor, 2001). Other species of Culicoides vectors may be implicated in BTV circulation (Mellor and Wittmann, 2002), such as Culicoides obsoletus, Culicoides pulicaris, 
Culicoides newsteadi and Culicoides circumscriptus. Indeed, BTV has been detected in C. obsoletus in Cyprus (Mellor and Pitzolis, 1979) and, more recently, in southern Italy (Savini et al., 2003). It has also been detected in C. pulicaris in Sicily (Caracappa et al., 2003). The presence of C. imicola has been recorded in many infected regions but surveillance has found this species to be abundant only in Sardinia and eastern Calabria (Goffredo et al., 2001). In Sicily (Italy), the first BT outbreak was detected on the 13 October 2000 in Palermo province (Caracappa et al., 2001), 76 outbreaks have been registered so far. The entomological surveillance has reported that $C$. imicola has never been particularly widespread there (Goffredo et al., 2001). This information, along with the isolation of BTV from other Culicoides species, suggests a potential role of novel vectors in BTV transmission. The aim of this study is to report the different species of Culicoides found in farms with BTV and to investigate on the role of new competent vectors. Clinical and serological evidence of BTV activity has suggested a National Surveillance Plan, based on European Union Commission Decision 138/2001/CE, which provides a serological and entomological survey. This surveillance programme has involved the regular monitoring of sentinel animals (seronegative cattle) and operation of collecting insects using suitable traps.

\section{Materials and Methods}

This study has been carried out from 2003 to 2008 in Sicily. Sicily is an island in the middle of the Mediterranean sea with peculiar geographical characteristics favouring the introduction and the spreading of Culicoides vectors responsible for BTV transmission. According to the National Surveillance Plain, the regional territory has been subdivided in $400 \mathrm{~km}^{2}$ areas with 58 seronegative cattle periodically checked by serological tests (O.M. 11.05.2001). Serological sampling was performed once a month. A total number of 100332 sera samples were tested. The collected blood samples from 'cattle sentry' were tested by a competitive ELISA [provided by Istituto Zooprofilattico Sperimentale Abruzzo e Molise in Teramo (IZS Teramo), sensitivity $=100 \%$, specificity $=99.3 \%]$, to detect BTV antibodies in ruminant sera. The serotyping of all the positive sera has been made by the CESME at the IZS Teramo, by virus neutralization test according to the OIE Manual. Moreover, entomological captures were also performed to investigate the presence of insect vectors belonging to Culicoides genus in farms with seroconversions. The number of the farms sampled was 321 and 581 captures were done. Farms chosen have had at least one seroconversion. Insects were captured by using black-light traps, activated for about two consecutive nights near cattle sheds, located outdoors, close to herds. Black-light traps were switched on $1 \mathrm{~h}$ before sunset and in the following morning the insects were gathered and transported to the laboratory in $90 \%$ ethanol. Insects belonging to the Culicoides genus were separated from other insects and counted under a binocular dissecting microscope. Culicoides spp. were identified by means of Wirth, Dyce and Peterson (1985), Wirth and Hubert (1989), Rawlings (1996), Meiswinkel and Baylis (1998), Downes and Wirth (1981), taxonomical keys. For each entomological capture, the total number of insects, Culicoides spp. and Culicoides imicola were calculated. Captures having at least one Culicoides spp. were considered positive. In analogy, captures having at least one midge belonging to C. imicola, C. obsoletus, C. pulicaris, C. newsteadi, C. circuscriptus, Culicoides gejgelensis, Culicoides paulae, Culicoides subfascipennis, Culicoides tauricus, Culicoides agathensis, Culicoides fascipennis, Culicoides

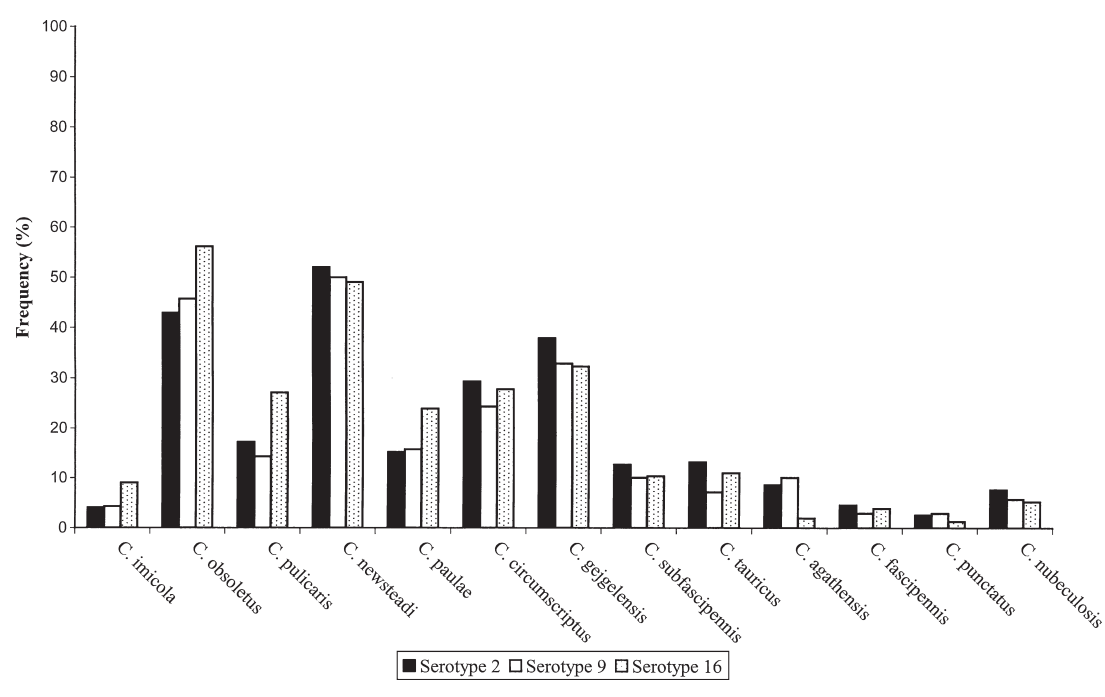

Fig. 1. Correlations between Culicoides species and BTV serotype. 
punctatus or Culicoides nubeculosis, were considered positive for those species.

\section{Results}

The BTV serotypes occurring from 2003 to 2008 in Sicily were primarily 2 and 16 (42\% and 53\%, respectively), followed by serotypes $9(3 \%)$ and $4(2 \%)$. The annual seroconversion rate found during the study was $1 \%$ in 2003, $4.07 \%$ in $2004,3.24 \%$ in $2005,1.34 \%$ in $2006,1.66 \%$ in 2007 and $0.2 \%$ in 2008, respectively. Concerning the entomological survey in BTV seropositive farms, we observed that $82 \%$ of checked farms were positive for $\mathrm{Cu}$ licoides spp. during the last 6 years, with considerable variability in the frequency of different species of Culicoides present. Figure 1 shows the correlations among the frequency of the different Culicoides species for each capture and the BTV serotypes 2, 9 and 16. Serotype 4 was excluded because only four positive farms were found to be infected by this serotype.

\section{Discussion}

The Bluetongue outbreak first occurred in Sardinia in 2000 and the virus rapidly spread to Sicily and to southern Italy (Anon, 2000). In Sicily, BTV-2 (Serotype 2) was first confirmed in October 2000. The source of the initial virus introduction remains uncertain, although illegal animal movement is suspected (Giovannini et al., 2004). The serological surveillance was improved according to the National Plan and the serologic control of imported animals have allowed to investigate the virus circulation and the introduction of new virus serotypes, such as serotype 1 (from Sardinia) and 8 (from Northern Europe). The entomological control enabled to produce detailed maps of different species of Culicoides circulating in Sicily and to evaluate the presence of competent BTV vectors.

From 2003 to 2008, we tested 100332 sera samples and we registered 1772 seroconversions. Since 2003, BTV serotype $(2,4,9,16)$ circulation remained unvaried and no new serotype was introduced. The most frequent serotypes are 2 and 16 . The rate of seroconversions has decreased after 2004. It is necessary to underline that from 2002, according to the National Surveillance Plan, receptive animals were vaccinated to the BTV serotypes 2, 4, 9 and 16. We cannot determine if seroconversions were caused by wild-type or vaccine virus. Referring to the entomological survey, we observed that only $10 \%$ of Culicoides spp. positive farms were positive for $C$. imicola. The small number of positive farms for C. imicola suggested to investigate, for each capture, the presence of other potential vectors to evaluate their role in the spreading BT disease. The most prevalent species observed were $C$. obsoletus and C. newsteadi (these species did not differ significantly in prevalence, $54.52 \%$ and $55.45 \%$, respectively), followed by C. gejgelensis (35.51\%), C. circumscriptus (31.78\%), C. pulicaris $(22.12 \%)$, C. paulae $(18.07 \%)$, C. subfascipennis (15.58\%), C. tauricus (11.84\%), C. agathensis (7.48\%), C. fascipennis and C. nubeculosis (5.61\%) and C. punctatus (3.12\%). Some other Culicoides species were found but their frequency was less than $1 \%$. Analysis of data collected do not detect any correlation between different BTV serotypes of and Culicoides species found (Fig. 1). Considering that we performed in total 581 captures in seropositive farms and we found C. imicola only in 70 captures, it may be supposed that other competent insect vectors are endemic in Sicily. Furthermore, we do not know if two-night catches are sufficient to give a significant picture of the distribution of vectors. Also, data reported are not sufficient to perform a statistical analysis between seroconversion and different Culicoides species, but the high frequency of $C$. pulicaris and $C$. obsoletus in seropositive farms suggests that in Sicily, these species may play a role in the transmission of BTV, as already reported in the literature (Mellor and Pitzolis, 1979; Mellor and Wittmann, 2002; Caracappa et al., 2003; Savini et al., 2003).

\section{References}

Anon, 2000: Bluetongue in Italy: follow-up report No. 1. Off. Int. Epiz. Dis. Inform. 13, 209-210.

Baylis, M., and P. S. Mellor, 2001: Bluetongue around the Mediterranean in 2001. Vet. Rec. 149, 659.

Caracappa, S., C. Di Bella, A. Guercio, F. Prato, and A. Torina, 2001: Emergenza Blue Tongue in Sicilia: Controllo del Territorio ed Attività di Sorveglianza, pp. 53-66. S.I.P.A.O.C., Perugia, 16 November 2001.

Caracappa, S., A. Torina, A. Guercio, F. Vitale, A. Calabrò, G. Purpari, V. Ferrantelli, M. Vitale, and P. S. Mellor, 2003: Identification of a novel bluetongue virus vector species of Culicoides in Sicily. Vet. Rec. 153, 71-74.

Downes, A., and W. W. Wirth, 1981: Ceratopogonidae. In: McAlpine, J. F., B. V. Peterson, G. E. Shewell, H. J. Teskey, J. R. Vockeroth, and D. M. Wood (eds), Manual of Nearctic Diptera. Volume 1, Chapter 28., pp. 393-421. Agriculture Canada Monograph 27.

Giovannini, A., P. Calistri, A. Conte, L. Savini, D. Nannini, D. Patta, U. Santucci, and V. Caporale, 2004: Bluetongue virus surveillance in a newly infected area. Vet. Ital. 40, 188-197.

Goffredo, M., G. Satta, A. Torina, G. Federico, P. Scaramozzino, M. A. Cafiero, R. Lelli, and R. MeiswinkeI, 2001: The 2000 Bluetongue Virus (BTV) Outbreak in Italy: Distribution and Abundance of the Principal Vector Culicoides Imicola Kieffer. Xth International Symposium of Veterinary Laboratory Diagnosticians and OIE Seminar on Biotechnology, pp. 308309. Salsomaggiore, Parma, Italy. 
Linton, Y. M., A. J. Mordue, R. H. Cruickshank, R. Meiswinkel, P. S. Mellor, and J. F. Dallas, 2002: Phylogenetic analysis of the mitochondrial cytochrome oxidase subunit I gene of five species of the Culicoides imicola species complex. Med. Vet. Entomol. 16, 139-146.

Meiswinkel, R., and M. Baylis, 1998: Morphological confirmation of the separate species status of Culicoides (Avaritia) nudipalpis Delfinado, 1961 and C. (A.) imicola Kieffer, 1913 (Diptera: Ceratopogonidae). Onderstepoort J. Vet. Res. 65, 9-16.

Mellor, P. S., and G. Pitzolis, 1979: Observations on breeding sites and light-trap collections of Culicoides during an outbreak of bluetongue in Cyprus. Bull. Entomol. Res. 69, 229-234.

Mellor, P. S., and E. J. Wittmann, 2002: Bluetongue virus zin the Mediterranean basin, 1998-2001. Vet. J. 164, 20-37.

Mellor, P. S., J. Boorman, and M. Baylis, 2000: Culicoides biting midges: their role as Arbovirus Vectors. Ann. Rev. Entomol. 45, 307-340.

Ministero della Sanità, Ordinanza del 11 Maggio. 2001: Misure urgenti di profilassi vaccinale obbligatoria contro la febbre catarrale degli ovini (Bluetongue). Disposizioni per lo spostamento degli animali. Allegato, 1, parte 4, $1-16$.

Rawlings, P., 1996: A key, based on wing patterns of biting midges (genus Culicoides Latreille-Diptera: Ceratopogonidae) in the Iberian Peninsula, for use in epidemiological studies. Graellsia 52, 57-71.

Savini, G., M. Goffredo, F. Monaco, P. De Santis, and R. Meiswinkel, 2003: Transmission of bluetongue virus in Italy. Vet. Rec. 152, 119.

Wirth, W. W., A. L. Dyce, and B. V. Peterson, 1985: An atlas of wing photographs, with a summary of the numerical characters of the Nearctic species of Culicoides (Diptera: Ceratopogonidae). Contrib. Am. Entomol. Inst. 22, 1-46.

Wirth, W. W., and A. A. Hubert, 1989: The Culicoides of Southeast Asia (Diptera: Ceratopogonidae). Mem. Amer. Entomol. Inst. 44, 1-508.

Wittmann, E. J., and M. Baylis, 2000: Climate change: effects on Culicoides-transmitted viruses and implications for the UK. Vet. J. 160, 107-117. 